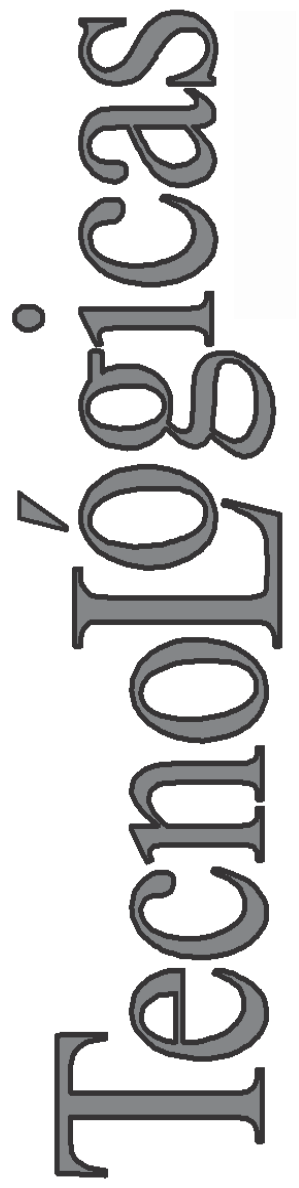

\title{
Algoritmo Genético Multiobjetivo para Enrutamiento con Calidad de Servicio en Redes Inalámbricas Mesh
}

\section{Multiobjective Genetic Algorithm for QoS Routing in Wireless Mesh Networks}

Wilson Soto $^{1}$ Amparo Olaya-Benavides ${ }^{2}$

1 Grupo de Investigación SIGA - Sistemas Inteligentes e Información Espacial, Universidad Central, Bogotá-Colombia wsoto@ucentral.edu.co

2 Grupo de Investigación SIGA - Sistemas Inteligentes e Información Espacial, Universidad Central, Bogotá-Colombia aolayab@ucentral.edu.co 


\title{
Resumen
}

Las redes inalámbricas mesh o en forma de malla son el tipo de redes más utilizadas actualmente, al igual que presentan interesantes desafíos en el área de investigación. Uno de estos desafíos es como mejorar la capacidad que tiene una red de proveer un mejor servicio seleccionando el tráfico de red sobre diversas tecnologías. Para ello se requiere tener en cuenta variables tales como el retardo, el promedio de retardo, la perdida de paquetes y el ancho de banda. Este tipo de desafío donde es necesario optimizar varias variables, se convierte en un problema combinatorio y computacional complejo. Uno de los métodos para solucionar este tipo de problemas son los algoritmos genéticos. En este trabajo se presenta un algoritmo genético multiobjetivo para el problema de calidad de servicio en enrutamiento de redes inalámbricas en forma de malla. El algoritmo genético propuesto además tiene la ventaja de usar el algoritmo de frontera de Pareto con la técnica divide y vencerás y el algoritmo de doblez para seleccionar una única solución del problema. Las rutas encontradas por el algoritmo propuesto demuestran que se cumple el propósito de mejorar la calidad de servicio en el enrutamiento de redes inalámbricas tipo malla.

\section{Palabras clave}

Redes inalámbricas mesh; calidad de servicio; enrutamiento; optimización multiobjetivo; algoritmo genético.

\begin{abstract}
The wireless mesh network is the type of network more used currently, the same as show interesting challenges in the topic of investigation. One of the challenges is as improve the capability of a network to provide better service to selected network traffic over various technologies. This requires keep in mind variables such as delay, jitter, packet loss and band width. This type of challenge where is necessary to optimize several variables becomes a combinatorial and computational complex problem. A method for solve this type of problems are the genetic algorithms. In this work we show a multiobjective genetic algorithm for quality of service in routing in wireless mesh networks. The proposed genetic algorithm besides has the advantage of use the Pareto frontier algorithm with the divide and conquer technique and the knee algorithm for to select an only solution of the problem. The paths found for the proposed algorithm demonstrate that achieve the purpose to improve the quality of service in routing in wireless mesh networks.
\end{abstract}

\section{Keywords}

Wireless mesh networks; quality of service; routing; multiobjective optimization; genetic algorithm. 


\section{INTRODUCCIÓN}

Una red inalámbrica mesh (WMN por su siglas en inglés Wireless Mesh Network) es una red con una topología en forma de malla. Uno de los aspectos más importantes a tener en cuenta en una red WMN es la calidad de servicio (QoS por sus siglas en ingles Quality of Service) definida por ITU-T (2008) como la totalidad de las características de un servicio de telecomunicaciones que determinan su capacidad para satisfacer las necesidades explícitas e implícitas del usuario del servicio. Para la medición de la QoS se involucran diferentes variables que tienen como propósito garantizar el funcionamiento de la red.

Las variables para medir QoS pueden ser representadas como objetivos dentro del problema. En este tipo de problemas los objetivos pueden estar en conflicto y cada objetivo tiene una solución diferente. Una forma de encontrar soluciones al problema es optimizar simultáneamente todos los objetivos, esto se denomina optimización multiobjetivo. Dentro del marco de éste tipo de soluciones existen diferentes métodos entre los que se destacan los algoritmos genéticos. La propuesta que se presenta en ése artículo corresponde a una solución de enrutamiento con QoS en redes WMN mediante algoritmos genéticos multiobjetivo.

\section{METODOLOGíA}

\subsection{Revisión de Trabajos Relacionados}

En la actualidad se han desarrollado diversos trabajos relacionados con la optimización multiobjetivo para el enrutamiento en redes: En (Gomes \& Huiban, 2007) proponen un algoritmo multiobjetivo con dos objetivos: el balance de carga en los enrutadores para incrementar la seguridad en caso de fallos y el minimizar el tiempo de comunicación. Esta solución utiliza la frontera de Pareto y el método de generación de columnas (método de optimización basado en el método simplex). Principalmente este trabajo fue orientado a resolver el problema RWP (en inglés Round Weighting Problem) el cual corresponde a la unión del problema de ruteo y el 
problema de programación para satisfacer una demanda que se encuentra sujeta a interferencias de múltiples accesos. El problema de la solución propuesta radica en el tamaño del problema, si este llega a ser demasiado complejo es decir un espacio de búsqueda de soluciones demasiado grande el problema se convierte en un problema polinomial no determinista (Camelo et al., 2010).

En (Crichigno et al., 2008) se propone un algoritmo con programación dinámica teniendo en cuenta dos objetivos: el primer objetivo medido como el número de bits por segundo que son enviados a lo largo de la ruta entre la fuente y el destino y el segundo objetivo el retardo o la latencia punto a punto para mejorar la calidad de servicio en el enrutamiento. Una de las debilidades de este tipo de soluciones está en la técnica de programación dinámica relacionada con el tamaño del problema, cuando el problema es muy grande el tiempo en la búsqueda de una solución óptima se incrementa polinómicamente.

Otro propuesta se encuentra en (Zhuo et al., 2010). En este trabajo se identifica el problema de enrutamiento como un problema polinomial no determinista NP y es transformado a un problema de optimización multiobjetivo. Los autores presentan un algoritmo de optimización de enjambre de partículas PSO (en inglés Particle Swarm Optimization) discreto para aproximarse a la frontera de Pareto y determinar el conjunto de soluciones óptimas o no dominadas teniendo en cuenta tres objetivos de calidad de servicio simultáneamente. Sin embargo, los resultados presentados después de hallar las soluciones óptimas o soluciones no dominadas de la frontera de Pareto no incluyen métricas para evaluar la calidad de las soluciones.

Otro trabajo que destaca la utilización de algoritmos evolutivos multiobjetivo para solucionar los problemas de enrutamiento con calidad de servicio en redes mesh en (Camelo et al., 2010). Los autores utilizan un algoritmo evolutivo multiobjetivo denominado NSGA-II. El modelo propuesto se trabaja con métricas como: ancho de banda, tasa de pérdida de paquetes, retardo (latencia) y poder de consumo. Este modelo de solución trabaja de manera óptima en ambientes de redes convergentes en los cuales los servicios que se despliegan en dicha red deben ser garantizados bajo un conjunto de parámetros de QoS. Aunque, los autores mencionan 
que la utilización del algoritmo NSGA-II cumplió con las expectativas del trabajo, el algoritmo NSGA-II no siempre obtiene los mejores resultados en la búsqueda de soluciones óptimas en problemas de optimización multiobjetivo (Soto et al., 2012). Además, el trabajo muestra una comparación del algoritmo NSGA-II con un algoritmo mono-objetivo, una debilidad para presentar resultados, discusiones o conclusiones más generales de la aplicación de los algoritmos multiobjetivo en la QoS en enrutamiento sobre WMN.

\subsection{Formulación Matemática}

Una red WMN es representada como grafo $G=(V, E)$, donde $V$ representa el conjunto de nodos dentro de la red y $E$ constituye el conjunto de enlaces entre cada uno de los nodos, $P$ representa la ruta entre el nodo $s(s \in V)$ y el nodo destino $d(d \in V)$, los índices $i$ y $j$ tal que $A=\{(i, j) \mid i, j \in V, i \neq j\}$ representan el conjunto de enlaces entre los nodos en forma de tuplas. Para un enlace $P$ se pueden definir los siguientes parámetros de QoS: delay (retardo), jitter (medida de la variación promedio del delay) y el ancho de banda. Formalmente el delay está definido en (1) y el jitter está definido en (2).

$$
\begin{aligned}
& \operatorname{delay}(p)=\sum_{e\left(v_{i}, v_{j}\right) \in E(p)} \operatorname{delay}\left(e\left(v_{i}, v_{j}\right)\right) \\
& \operatorname{jitter}(p)=\sum_{e\left(v_{i}, v_{j}\right) \in E(p)} \operatorname{jitter}\left(e\left(v_{i}, v_{j}\right)\right)
\end{aligned}
$$

\subsection{Algoritmo Propuesto}

En este trabajo se propone un algoritmo genético multiobjetivo (Algoritmo 1) para buscar las rutas que existen entre dos nodos en una red. Las rutas encontradas consideran de forma simultánea los valores mínimos para el delay y el jitter teniendo en cuenta un valor máximo de ancho de banda.

Las rutas encontradas corresponden a un conjunto de soluciones que representan valores de equilibrio entre los objetivos selec- 
cionados, que para el problema son el delay y el jitter. Estos tipos de problemas que buscan soluciones teniendo en cuenta más de un objetivo son llamados problemas multiobjetivo y las propuestas para solucionarlos son comúnmente metaheurísticas como los algoritmos genéticos (Donoso y Fabregat, 2007). Además, estas propuestas utilizan la técnica de la frontera de Pareto (Zitzler et al., 2004) para encontrar el conjunto de soluciones al problema.

Los algoritmos genéticos son algoritmos basados en mecanismos biológicos como las leyes de Mendel y el principio de selección natural de Darwin. Típicamente un algoritmo genético está compuesto por: Los individuos son elementos que representan la solución al problema. En este caso los individuos son las rutas validas que existen en la red aleatoria (Algoritmo 1 - Líneas 1 y 3). Cada gen del individuo representa un nodo dentro de la ruta. El conjunto de individuos es llamado población $(P)$.

La reproducción es la etapa de combinación de los cromosomas. El cruce (Algoritmo 1 - Línea 5) y la mutación (Algoritmo 1 - Línea 11) son métodos de reproducción. El cruce es de tipo de punto único y la mutación consiste en seleccionar un número de genes aleatorios del individuo para ser cambiados. El índice de calidad o fitness es una medida abstracta para clasificar los individuos. En este problema el fitness (Algoritmo 1 - Líneas 8, 10 y 13) para cualquier individuo válido es determinado por el objetivo 1 (delay) y el objetivo 2 (jitter) y que cumpla la restricción $(R)$ de ancho de banda. La etapa de selección consiste en determinar los individuos que sobreviven para la próxima generación. El algoritmo propuesto utiliza la técnica de frontera de Pareto (Algoritmo 1 - Línea 14) sobre los individuos, seleccionando aquellos que tiene mínimos valores en los objetivos y un valor que satisface la restricción (3).

$\forall$ ind $\in P, \operatorname{ind}_{o b j 1} \leq \min _{o b j 1} \wedge \operatorname{ind}_{o b j 2} \leq \min _{o b j 2} \wedge \operatorname{ind}_{R} \leq R$

\section{RESULTADOS Y DISCUSIÓN}

El primer experimento se realizó con una red virtual de 10 nodos. La Tabla 1 muestra los arcos entre los nodos con sus respectivos los valores para cada uno de los objetivos y la restricción. Se 
tomó como nodo inicial el nodo 1 y como nodo final el nodo número 10.

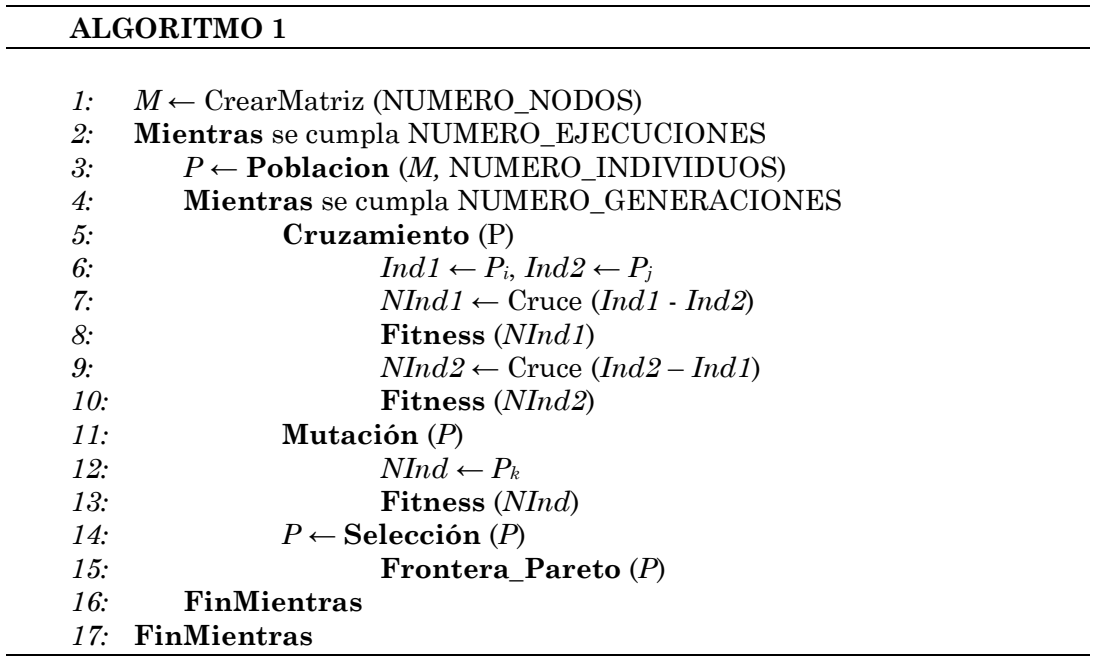

Los parámetros de ejecución del algoritmo genético fueron una población de 100 individuos, 500 generaciones, una tasa de cruce del $25 \%$ y una tasa de mutación del $25 \%$.

Los resultados del primer experimento se pueden ver en la Fig. 1 donde aparecen las soluciones representadas como puntos dibujados en dos coordenadas. Las soluciones son las diferentes rutas encontradas por el algoritmo y las coordenadas son los objetivos para el problema. Se puede observar en la figura la frontera de Pareto para el conjunto de puntos, es decir, el mejor conjunto de soluciones encontradas para el problema con los parámetros de ejecución del algoritmo. De igual forma la figura muestra la representación de la red virtual de 10 nodos con todas las posibles rutas, las mejores soluciones encontradas y la solución seleccionada.

En la Tabla 2 se muestra con detalle las rutas y los valores mínimos para cada solución del conjunto de la frontera de Pareto. La solución marcada corresponde a una única solución seleccionada utilizando el algoritmo de doblez (Branke et al. 2004) que mediante ángulos entre las soluciones de la frontera de Pareto determina una única solución. 
Tabla 1. En cada celda aparecen en su orden los valores que representan la restricción, objetivo1 y objetivo2 entre el nodo $i$ y el nodo $j$. Fuente: Autores

\begin{tabular}{|c|c|c|c|c|c|c|c|c|c|c|}
\hline & 1 & 2 & 3 & 4 & 5 & 6 & 7 & 8 & 9 & 10 \\
\hline \multicolumn{11}{|l|}{1} \\
\hline & 0.904 & & & & & & & & & \\
\hline \multirow[t]{3}{*}{2} & 74 & & & & & & & & & \\
\hline & 0.681 & & & & & & & & & \\
\hline & 0,480 & 0,258 & & & & & & & & \\
\hline \multirow[t]{3}{*}{3} & 50 & 68 & & & & & & & & \\
\hline & 0,205 & 0,811 & & & & & & & & \\
\hline & - & 0,063 & 0,767 & & & & & & & \\
\hline \multirow[t]{3}{*}{4} & - & 65 & 24 & & & & & & & \\
\hline & - & 0,459 & 0,206 & & & & & & & \\
\hline & 0,355 & 0,169 & - & 0.591 & & & & & & \\
\hline \multirow[t]{3}{*}{5} & 12 & 46 & - & 85 & & & & & & \\
\hline & 0,244 & 0,067 & - & 0.344 & & & & & & \\
\hline & 0,424 & 0.256 & - & 0,539 & - & & & & & \\
\hline \multirow[t]{3}{*}{6} & 28 & 3 & - & 94 & - & & & & & \\
\hline & 0,734 & 0.271 & - & 0,466 & - & & & & & \\
\hline & - & 0.395 & - & 0.417 & - & 0,033 & & & & \\
\hline \multirow[t]{3}{*}{7} & - & 72 & - & 41 & - & 38 & & & & \\
\hline & - & 0.228 & - & 0.626 & - & 0,902 & & & & \\
\hline & - & 0,967 & - & - & 0.624 & 0,805 & 0.311 & & & \\
\hline \multirow[t]{3}{*}{8} & - & 15 & - & - & 14 & 58 & 15 & & & \\
\hline & - & 0,885 & - & - & 0.754 & 0,560 & 188 & & & \\
\hline & - & 0,994 & - & - & - & - & - & 0,019 & & \\
\hline \multirow[t]{3}{*}{9} & - & 91 & - & - & - & - & - & 75 & & \\
\hline & - & 0,297 & - & - & - & - & - & 0.541 & & \\
\hline & 0.945 & 0.941 & 0,657 & 0,008 & 0.312 & - & 0,008 & 0.123 & - & \\
\hline \multirow[t]{2}{*}{10} & 96 & 10 & 60 & 97 & 89 & - & 65 & 37 & - & \\
\hline & 0.934 & 0.956 & 0,056 & 0,415 & 0.344 & - & 0,568 & 0.524 & - & \\
\hline
\end{tabular}

Otra serie de experimentos llevados a cabo se resumen en la Tabla 3, donde, además de los valores de los objetivos para la única solución o solución balance, aparecen la cantidad de soluciones de la frontera de Pareto y el número de saltos o la cantidad de arcos que la ruta tiene para llegar desde el nodo inicial al nodo destino. Los parámetros de ejecución del algoritmo fueron 100 individuos, 100 generaciones, una tasa de cruce del $20 \%$, una tasa de mutación del 25\% y 10 ejecuciones diferentes. Además, el valor 
máximo de restricción para los individuos fue de 2.0, que para el problema en particular representa el ancho de banda.
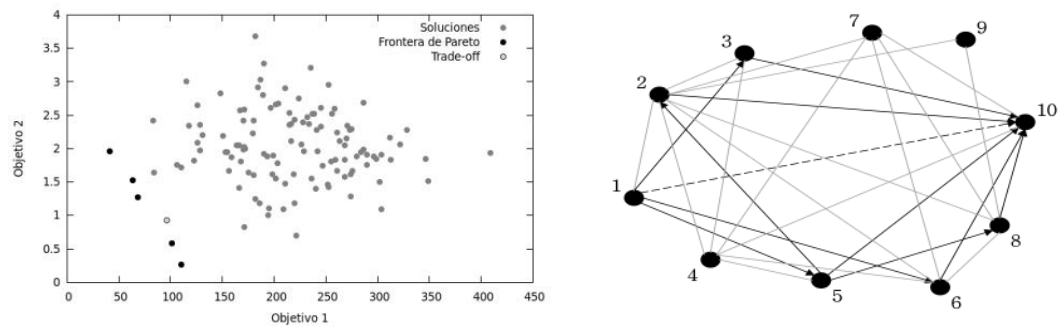

Fig. 1. A la izquierda las soluciones encontradas y a la derecha la representación de la red virtual con 10 nodos. Las líneas y puntos de color negro corresponden a la representación de la frontera de Pareto, es decir, las mejores soluciones encontradas que contienen los valores mínimos para el objetivo1, el objetivo2 y satisfacen la restricción. La línea punteada es la solución seleccionada que es representada por el punto blanco en la frontera de Pareto. Fuente: Autores

Tabla 2. Rutas encontradas para el problema de la Tabla 1 con sus respectivos valores para el objetivo1 y objetivo2, que representan la frontera de Pareto. En color gris se demarca la solución única o el trade-off de las soluciones. Fuente:

\begin{tabular}{ccc}
\multicolumn{3}{c}{ Autores } \\
\hline Ruta & Objetivo1 & Objetivo 2 \\
\hline$[1,3,10]$ & 110 & 0.2615828 \\
{$[1,5,10]$} & 101 & 0.5881632 \\
{$[1,10]$} & 96 & 0.9336746 \\
{$[1,5,2,10]$} & 68 & 1.2669900 \\
{$[1,5,8,10]$} & 63 & 1.5227494 \\
{$[1,6,2,10]$} & 41 & 1.9614249 \\
\hline
\end{tabular}

Basado en los resultados experimentales se puede poner en discusión varios hechos: Existe un promedio de soluciones encontradas (conjunto de frontera de Pareto) alrededor del 10\% que garantizan QoS con respecto a la cantidad de nodos y el número de conexiones de las redes virtuales usadas en los experimentos.

Los valores para la desviación estándar de los objetivos garantizan que la solución única que pertenece al conjunto de la frontera de Pareto puede ser diversa, es decir, el algoritmo puede encontrar varios enrutamientos con $\mathrm{QoS}$ donde los valores para las variables 
del delay y del jitter son muy diferentes. Se puede observar que las rutas únicas seleccionadas de la frontera de Pareto tienden a tener un mínimo número de saltos, lo que puede suponer que la QoS en el enrutamiento mejore en relación con otras variables como la perdida de paquetes.

Tabla.3. Resumen de los experimentos realizados a redes aleatorias de 33, 66 y 100 nodos. Aparece la cantidad de posibles soluciones que corresponden a la frontera de Pareto (FP)

\begin{tabular}{|c|c|c|c|c|c|c|c|c|c|c|c|c|}
\hline \multicolumn{5}{|c|}{33 Nodos } & \multicolumn{4}{|c|}{66 Nodos } & \multicolumn{4}{|c|}{100 Nodos } \\
\hline 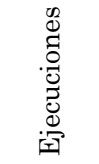 & FP & 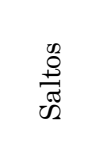 & 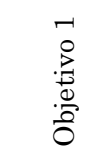 & 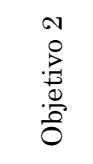 & $\mathrm{FP}$ & 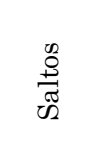 & $\begin{array}{l}\overrightarrow{0} \\
\stackrel{0}{0} \\
. \frac{0}{0} \\
0\end{array}$ & 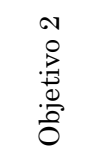 & $\mathrm{FP}$ & 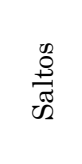 & 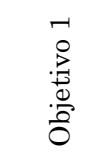 & $\begin{array}{l}N \\
\stackrel{0}{0} \\
\stackrel{0}{0} \\
\stackrel{0}{0} \\
0\end{array}$ \\
\hline 1 & 8 & 3 & 61,0 & 1,180 & 6 & 1 & 78,0 & 0,854 & 6 & 4 & 60,0 & 0,676 \\
\hline 2 & 7 & 3 & 61,0 & 1,180 & 6 & 2 & 81,0 & 0,360 & 8 & 2 & 100,0 & 0,432 \\
\hline 3 & 10 & 3 & 61,0 & 1,180 & 8 & 3 & 79,0 & 0,544 & 8 & 3 & 57,0 & 0,320 \\
\hline 4 & 7 & 3 & 61,0 & 1,180 & 7 & 1 & 78,0 & 0,854 & 5 & 2 & 50,0 & 0,783 \\
\hline 5 & 7 & 2 & 61,0 & 1,289 & 6 & 2 & 81,0 & 0,360 & 5 & 2 & 58,0 & 0,715 \\
\hline 6 & 8 & 2 & 61,0 & 1,289 & 7 & 1 & 78,0 & 0,854 & 5 & 2 & 58,0 & 0,715 \\
\hline 7 & 9 & 3 & 112,0 & 1,078 & 7 & 2 & 81,0 & 0,360 & 9 & 3 & 54,0 & 0,618 \\
\hline 8 & 7 & 3 & 29,0 & 1,446 & 5 & 1 & 78,0 & 0,854 & 7 & 3 & 82,0 & 0,694 \\
\hline 9 & 6 & 2 & 61,0 & 1,289 & 6 & 3 & 48,0 & 0,633 & 4 & 2 & 50,0 & 0,783 \\
\hline 10 & 8 & 3 & 61,0 & 1,180 & 6 & 3 & 48,0 & 0,633 & 7 & 3 & 54,0 & 0,618 \\
\hline Media & 7,624 & 2,656 & 60,176 & 1,225 & 6,351 & 1,712 & 71,679 & 0,593 & 6,205 & 2,521 & 60,777 & 0,615 \\
\hline $\begin{array}{l}\text { Dev. } \\
\text { Est. }\end{array}$ & 1,160 & 0,483 & 19,969 & 0,101 & 0,843 & 0,876 & 13,241 & 0,218 & 1,647 & 0,699 & 16,056 & 0,150 \\
\hline
\end{tabular}

\section{CONCLUSIONES}

En este trabajo se presentó un algoritmo genético para solucionar el problema de QoS de enrutamiento en redes mesh. El problema fue orientado a un tipo de problema multiobjetivo donde algunas de las variables que intervienen en la QoS en enrutamiento fueron elegidas como objetivos. Basado en los resultados del 
algoritmo genético propuesto y presentados en este trabajo, en los cuales se obtienen rutas en una red con un mínimo de delay, jitter, número de saltos y sin sobrepasar el ancho de banda máximo, se puede recomendar esta aproximación para garantizar QoS para enrutamiento de redes inalámbricas. Como trabajo futuro se pretende adicionar más objetivos, incluir métricas como la distancia, la distribución y la extensión que determinen la calidad de las soluciones que se encuentran en la frontera de Pareto y proponer otro tipo de metaheurísticas para solucionar el problema.

\section{AGRADECIMIENTOS}

Este trabajo fue financiado por el proyecto: "Calidad de servicio en enrutamiento de redes inalámbricas mesh usando algoritmos evolutivos multiobjetivo" aprobado por la convocatoria interna de investigación No. 2 de la Universidad Central - Colombia.

\section{REFERENCIAS}

Akyldiz Ian F. and Dr Xudong Wang.(2009). Wireless Mesh Networks. Ed. A Jon Wiley and Sons, Ltd. ISBN: 978-0470-03256-5.

Branke, J., Deb, K., Dierolf, H., and Osswald, M. (2004). Finding knees in multi-objective optimization. In Proceedings of the Parallel Problem Solving from Nature, LNCS, 3242, 2004, 722--731.

Camelo M., Omaña C. and Castro H. (2010). QoS routing algorithm based on multi-objective optimization for Wireless Mesh Networks. Communications (LATINCOM) IEEE Latin-American Conference, Septiembre 2010. pp. 875-881.

Donoso, Y., and Fabregat Ramon (2007). Multi-Objective Optimization in Computer Networks Using Metaheuristics.

Crichigno J., Khoury J., Wu M.Y. and Shu W. (2008), A Dynamic Programming Approach for Routing in Wireless Mesh Networks., in 'GLOBECOM' , IEEE, pp. 528-532.

Gomes C. and Huiban G.(2007). Multiobjective Analysis in Wireless Mesh Networks. In 15th Annual Meeting of the IEEE International Symposium on Modeling, Analysis, and Simulation of Computer and Telecommunication Systems-MASCOTS, Istanbul, Turkey, October 2007. pp. 103-108. 
ITU-T. International Telecommunication Union. (2008) E.800. Serie E: Explotación general de la red, servicio telefónico, explotación del servicio y factores humanos. Definiciones de términos relativos a la calidad del servicio.

Soto Daniel, Soto Wilson y Pinzón Yoan. (2012) Algoritmo de Optimización de Colonia de Hormigas Multi-Objetivo aplicado al problema de la mochila multidimensional. Revista Programación Matemática y Software 3(2): 20-31. ISSN: 2007-3283.

Zhuo Jun, Chen Yu-Zhong and Chen Yi-Ping. (2010). A novel multiobjective optimization algorithm based on discrete PSO for QoS multicast routing in wireless mesh networks. On 2010 International Symposium Intelligent Signal Processing and Communication Systems (ISPACS), China, December 2010.

Zitzler E., M. Laumanns, and S. Bleuler.(2004) A Tutorial on Evolutionary Multiobjective Optimization. In X. Gandibleux and others, editors, Metaheuristics for Multiobjective Optimisation, Lecture Notes in Economics and Mathematical Systems. Springer. 